Bull. Korean Math. Soc. 51 (2014), No. 6, pp. 1791-1804

http://dx.doi.org/10.4134/BKMS.2014.51.6.1791

\title{
COMMODITY FUTURES TERM STRUCTURE MODEL
}

\author{
Hyeong In Choi, Song-Hwa Kwon, Jun Yeol Kim, and Du-Seop Jung
}

\begin{abstract}
A new approach to the commodity futures term structure model is introduced. The most salient feature of this model is that, once the interest rate model is given, the commodity futures price volatility is the only quantity that completely determines the model. As a consequence this model enables one to do away with the drudgeries of having to deal with the convenience yield altogether, which has been the most thorny point so far.
\end{abstract}

\section{Introduction}

Modelling the commodity futures price has been a notoriously elusive task. The reason is that unlike its purely financial counterpart that has only to relate the spot price with the cost of capital, namely, the interest rate, the commodity futures price model must also take into account various costs and gains arising from the peculiarities of handling the physical asset such as storage, insurance and transportation costs, and the loss or gain incurred in securing the future delivery of the commodity. All these extraneous costs and gains are usually dubbed as the convenience yield. The trouble is that this convenience yield is not a quantity that can be readily observed per se in the market.

Nonetheless most authors traditionally treat the convenience yield as one of the primary quantities that constitute the building block of the commodity futures price model. So a typical approach goes as follows: one sets up a dynamic model for the convenience yield and also a dynamic model for the spot price from which, together with the interest rate model, the dynamics of the commodity futures price is constructed. The trouble with this approach is how to calibrate various parameters associated with the model, especially those governing the dynamics of the convenience yield. As the convenience yield

Received November 18, 2013; Revised May 5, 2014.

2010 Mathematics Subject Classification. Primary 60H30, 91G80.

Key words and phrases. commodity futures, term structure, convenience yield, volatility, European option, HJM.

The first author holds joint appointment with the Research Institute of Mathematics, Seoul National University. This research is supported by WCU-SNU 2009.

The second author was supported by the Catholic University of Korea, Research Fund, 2013.

(C)2014 Korean Mathematical Society 
cannot be directly observed in the market as an isolated quantity, the parameter of its dynamic model must be estimated somehow indirectly from the market data or construed from the totality of the commodity futures price model with the aid of various derived quantities. In either case, many difficulties, practical as well as theoretical, arise that must be carefully addressed, which add to the complexity and unrealism of the model.

The most well known models along this line are the ones proposed by Schwartz and his coauthors. In particular the models proposed by Gibson and Schwartz [12], Schwartz [23], and Miltersen and Schwartz [18] are the most well known. Although the details in there are different, all these models more or less follow the broad outline above. They also involve various other parameters that have to be calibrated, which is a nontrivial task for which Schwartz and his coauthors devised many ingenious methods like constructing auxiliary equations, measurement equation or transition equation, employing Kalman filter method, and so on.

Later, Schwartz and Smith [24] introduced a model in which the log spot price is split into two parts:

$$
\ln S(t)=\chi_{t}+\xi_{t}
$$

and constituent $\chi_{t}$ and $\xi_{t}$ are modelled separately, where the parameter estimation is similar to the earlier models. They also showed that this model is equivalent to the Gibson-Schwartz model [12].

Other commodity futures price models that involve stochastic processes of commodity spot price, the interest rate, and the convenience yield are [5, 14]. Some authors developed commodity futures theory with the specific property of commodities, such as the mean reversion property [2, 6, 20]. See also papers $[10,16]$. Some also added jump conditions [8, 11].

Many authors developed the theory of commodity futures from a historical (empirical) perspective. For instance, it is a generally observed phenomenon that the volatility and the price level have a high correlation. Many have developed the models to this effect $[14,21,22,26]$.

Others focus their attention on some specific aspects like the seasonal property of agriculture commodity futures [4, 17, 25].

There are other papers worth noticing. For instance, Anderson obtained some useful results concerning markovian term-structure model of commodity futures [1], and Casassus et al. suggested the concept of "maximal model" and "general equilibrium model" [6, 7]. And the papers [16, 19] looking at the relations between volatility and backwardation phenomenon of commodity futures price are also worth noticing.

However, the most noteworthy of all these models is the one proposed by Miltersen and Schwartz [18]. This model is different from others in that various quantities are postulated as having term structures, which gives added flexibility. It is also intimately related to our current work. 
Before we delving into further discussions, let us fix some notations and recall some basic facts.

Let $(\Omega, \mathcal{F}, P)$ be the usual probability space and let $\left\{\mathcal{F}_{t}\right\}_{t \geq 0}$ the usual filtration. One typically takes $\left\{\mathcal{F}_{t}\right\}_{t \geq 0}$ as the filtration generated by the Brownian motion $\mathbf{W}_{t}^{Q}$ and $\mathcal{F}=\bigvee \mathcal{F}_{t}$, which is the convention we use in this paper. We let $Q$ denote the martingale pricing measure which is assumed to exist and is used throughout this paper. $\mathbf{W}_{t}^{Q}$ is an $\mathcal{F}_{t}$-adapted Brownian motion under this martingale measure $Q$ with values in $\mathbb{R}^{d}$. Let $F(t, T)$ be the price at time $t$ of a commodity futures contract that delivers the commodity at time $T$ and let $S(t)=F(t, t)$ be its spot price at $t$. Since the futures contract itself is a tradable quantity with no carrying cost, the general theory tells us that it must be of the form

$$
F(t, T)=\mathbb{E}^{Q}\left[S_{T} \mid \mathcal{F}_{t}\right] .^{1}
$$

The basic building block of the Miltersen-Schwartz model consists of three stochastic processes: the spot price, the forward interest rate of the HJM model introduced by Heath, Jarrow and Morton [13] and the term structure of the convenience yield. They also assume that the futures price $F(t, T)$ is of the form

$$
F(t, T)=S_{t} \exp \left(\int_{t}^{T}(f(t, s)-\epsilon(t, s)) d s\right),
$$

where $f(t, T)$ is the usual forward interest rate of the HJM model and $\epsilon(t, T)$ is the term structure of the convenience yield.

The three basic building blocks are assumed to satisfy the following:

(A) (instantaneous forward rate model: HJM model)

$$
d f(t, s)=\mu_{f}(t, s) d t+\boldsymbol{\sigma}_{f}(t, s) \cdot d \mathbf{W}_{t}^{Q}
$$

(B) (convenience yield model)

$$
d \epsilon(t, s)=\mu_{\epsilon}(t, s) d t+\boldsymbol{\sigma}_{\epsilon}(t, s) \cdot d \mathbf{W}_{t}^{Q}
$$

(C) (spot model)

$$
d S(t)=\mu_{s}(t) S_{t} d t+S_{t} \boldsymbol{\sigma}_{s}(t) \cdot d \mathbf{W}_{t}^{Q} .
$$

Moreover, Miltersen and Schwartz showed that this model reverts back to the earlier model in [23] if one assumes volatilities of convenience yield and instantaneous forward rate are of a certain specific form. In particular if one assumes that the volatilities $\sigma_{\epsilon}(t, T)$ and $\sigma_{f}(t, T)$ of convenience yield and instantaneous forward rate, respectively, are of the form $\sigma_{\epsilon}(t, T)=\sigma_{\epsilon} e^{-\kappa_{\epsilon}(T-t)}$, $\sigma_{f}(t, T)=\sigma_{f} e^{-\kappa_{f}(T-t)}$, then the dynamics of $\epsilon(t, t)$ and $f(t, t)$ follow certain type of Ornstein-Uhlenbeck process, which is the one dubbed as Schwartz III in [23]. This argument is analogue to getting the Hull-White model from the

\footnotetext{
${ }^{1}$ In [18], they use $G(t, T)$ for the futures price, while we use $F(t, T)$. We interchangeably use $S(t)$ and $S_{t} ; F(t, T)$ and $F_{t}^{T}$ are also used interchangeably throughout this paper.
} 
HJM formulation by setting the diffusion term to be of form $\sigma e^{-a(T-t)}$. For details, see [3].

However, in a surprising turn, we discovered that it is possible to devise a model that enables one to do away with the drudgeries of having to deal with the convenience yield altogether. It turns out that our model is very much akin to that of Miltersen and Schwartz. In fact, the ingredients we use and the key facts derived therefrom are known to them. The key difference is that we do away with the spot price model and instead model directly the futures price $F(t, T)$ itself as the primary building block; and demote the convenience yield as a dependent (i.e., entirely derivable from the model) quantity. This change of viewpoint has quite surprising consequences in that it makes our model practical and easy to use. Moreover, the parameters that have to be calibrated in our model are minimal and they are all "volatility"-type constants, which are easiest to estimate; and there is nothing more.

Let us now describe our model. We first postulate, as Miltersen and Schwartz do, that the commodity futures price is of the form:

$$
F(t, T)=S(t) \exp \left(\int_{t}^{T} h(t, u) d u\right)
$$

for some quantity $h(t, T)$, which is the same as (1).

Let $f(t, T)$ be the forward interest rate in the HJM model. Then it is well known that the bond price $p(t, T)$ is written as:

$$
p(t, T)=\exp \left(-\int_{t}^{T} f(t, u) d u\right) .
$$

Now let $g(t, T)=h(t, T)-f(t, T)$. Thus $g(t, T)$ is the negative of $\epsilon(t, T)$ of [18]. It is also well known that, the commodity futures prices have complicated "term structure" behaviors like contango or backwardation, which typically do not appear in the futures of financial assets or contracts like stock index, bond or currency. This $g(t, T)$, hence $h(t, T)$, is a device to accommodate in the model such phenomena or contingencies.

Let us see why (2) is a reasonable form for the commodity futures price. Since $F(t, T)$ is a martingale under the martingale measure $Q$, we can set up the dynamics of $F(t, T)$ as satisfying the following stochastic differential equation:

$$
d F(t, T)=F(t, T) \boldsymbol{\sigma}(t, T) \cdot d \mathbf{W}_{t}^{Q}
$$

for some $\mathcal{F}_{t}$ adapted process $\sigma(t, T)$. This is due to the martingale representation theorem. Then since $S(t)=F(t, t)$, the spot dynamics is

$$
\begin{aligned}
d S(t) & =d F(t, t)+\left.\frac{\partial F}{\partial T}(t, T) d t\right|_{T=t} \\
& =F(t, t) \boldsymbol{\sigma}(t, t) \cdot d \mathbf{W}_{t}^{Q}+\frac{\partial F}{\partial T}(t, t) d t
\end{aligned}
$$




$$
=S(t)\left(\frac{1}{S(t)} \frac{\partial F}{\partial T}(t, t) d t+\boldsymbol{\sigma}(t, t) \cdot d \mathbf{W}_{t}^{Q}\right) .
$$

Define

$$
h(t, t)=\frac{1}{S(t)} \frac{\partial F}{\partial T}(t, t)=\frac{1}{F(t, t)} \frac{\partial F}{\partial T}(t, t) .
$$

This suggests that it is reasonable to define $h(t, T)$ as of the form

$$
h(t, T)=\frac{1}{F(t, T)} \frac{\partial F}{\partial T}(t, T) .
$$

Then upon integrating above equation with respect to variable $T$ on time interval $[t, T]$, we get the fundamental form of futures price process as

$$
F(t, T)=F(t, t) \exp \left(\int_{t}^{T} h(t, u) d u\right)=S(t) \exp \left(\int_{t}^{T} h(t, u) d u\right) .
$$

There is one issue arising from this representation: namely, the presence in (3) of the spot price $S(t)$ in the expression of the commodity futures price $F(t, T)$. This may look contradictory to our claim that our model does not involve the spot price as one of the primary building blocks. While (3) may look that way, we will show below that in our model the spot price is indeed only a derived quantity that does not enter into our model in any essential manner. Note that for most commodities the most liquid market is the futures market and the spot market is a rather thinly traded one. This is why we prefer not to rely on the spot price in our model.

\section{The model}

In this section, we formally present our model.

Before we proceed, let us agree on the following convention: the bold-face letter such as $\boldsymbol{\sigma}$ always stands for a vectorial quantity with values in $\mathbb{R}^{d}$; the dot between two vectorial quantities denotes the dot (inner) product.

The commodity futures is intricately tied with the interest rate. Throughout this paper we use the following HJM model [13] for the forward interest rate $f(t, T)$ satisfying

$$
d f(t, T)=\mu_{f}(t, T) d t+\sigma_{f}(t, T) \cdot d \mathbf{W}_{t}^{Q},
$$

where $\mu_{f}(t, T)$ is an $\mathbb{R}$-valued $\mathcal{F}_{t}$ adapted process and $\boldsymbol{\sigma}_{f}(t, T)$ is an $\mathbb{R}^{d}$-valued $\mathcal{F}_{t}$ adapted process called the forward (interest) rate volatility. We assume that $\mu_{f}(t, T)$ and $\boldsymbol{\sigma}_{f}(t, T)$ satisfy the standard assumptions of the HJM model [3].

As we shall see below, the description of the commodity futures price $F(t, T)$ involves a certain semi-martingale. (Say, for instance, $h(t, T)$ in the Model below.) We need to pin down its drift and volatility terms as follows. 
Definition. Let $\Delta=\left\{((t, \omega), T) \mid t, T \in \mathbb{R}^{+}, 0 \leq t \leq T, \omega \in \Omega\right\}$ and let $\phi$ be a stochastic process parametrized by $T$ given by

$$
\phi:((t, \omega), T) \in \Delta \mapsto \phi(t, T)(\omega) \in \mathbb{R}^{d} .
$$

(We usually suppress $\omega$ and use $\phi(t, T)$ instead.) $\phi(t, T)$ is called a drift process if it satisfies the followings:

(i) $\phi$ is $\mathcal{P} \times\left.\mathcal{B}^{+}\right|_{\Delta}$ measurable, where $\mathcal{P} \times\left.\mathcal{B}^{+}\right|_{\Delta}$ is the restriction to $\Delta$ of $\mathcal{P} \times \mathcal{B}^{+}$while $\mathcal{P}$ is the predictable $\sigma$-field and $\mathcal{B}^{+}$is the Borel $\sigma$-field of $\mathbb{R}^{+}$;

(ii) for all $t \leq T$ and $\omega \in \Omega, \phi(t, T)$ is uniformly bounded.

$\phi(t, T)$ is called a volatility process if it satisfies, besides (i) and (ii) above, the following:

(iii) for any $T$ and $t \leq T,(\omega, T) \mapsto \int_{0}^{t} \phi(s, T) \cdot d \mathbf{W}_{s}^{Q}$ is $\mathcal{F} \times \mathcal{B}^{+}$measurable.

These conditions are spelled out to make sure that $F(t, T)$ proposed below is well-defined and that various differentiation operations and the stochastic Fubini theorem work. In practice, however, simpler assumptions will do; namely, the whole thing can be made to work by assuming $\phi(t, T)$ is uniformly bounded and pathwise continuous in both variables $t$ and $T$ together with some mild measurability conditions. We are now ready to state our model.

\section{The Model}

(I) (Structural form of $F(t, T)$ ) The commodity futures price $F(t, T)$ is of the form:

$$
F(t, T)=S(t) \exp \left(\int_{t}^{T} h(t, u) d u\right),
$$

where $S(t)=F(t, t)$ is the spot price and $h(t, T)$ is given by

$$
d h(t, T)=\mu_{h}(t, T) d t+\boldsymbol{\sigma}_{h}(t, T) \cdot d \mathbf{W}_{t}^{Q}
$$

for some $\mathbb{R}$-valued drift process $\mu_{h}(t, T)$ and $\mathbb{R}^{d}$-valued volatility process $\boldsymbol{\sigma}_{h}(t, T)$;

(II) (Dynamics of $F(t, T)$ ) The futures price satisfies

$$
d F(t, T)=F(t, T) \boldsymbol{\sigma}(t, T) \cdot d \mathbf{W}_{t}^{Q}
$$

for some $\mathbb{R}^{d}$-valued volatility process $\boldsymbol{\sigma}(t, T)$ which is called the commodity futures volatility; moreover, for each $t$ and $\omega \in \Omega, \boldsymbol{\sigma}(t, T)$ is differentiable with respect to $T$ except possibly at finitely many points and its $T$-derivative, $\frac{\partial}{\partial T} \boldsymbol{\sigma}(t, T)$, wherever defined, is uniformly bounded;

(III) (Constituents of $h(t, T)) h(t, T)$ is the sum of $g(t, T)$ and the instantaneous forward rate $f(t, T)$; i.e.,

$$
h(t, T)=f(t, T)+g(t, T),
$$


where $g(t, T)$ satisfies

$$
d g(t, T)=\mu_{g}(t, T) d t+\boldsymbol{\sigma}_{g}(t, T) \cdot d \mathbf{W}_{t}^{Q}
$$

for some $\mathbb{R}$-valued drift process $\mu_{g}(t, T)$ and $\mathbb{R}^{d}$-valued volatility process $\boldsymbol{\sigma}_{g}(t, T)$.

Remark 2.1. This Model looks quite complicated involving a mouthful of quantities. However, we will show below that given the HJM forward rate volatility $\boldsymbol{\sigma}_{f}(t, T)$, or alternatively the volatility of the bond price dynamics, the whole thing simply boils down to the single quantity $\boldsymbol{\sigma}(t, T)$, the commodity futures price volatility, from which all other parameters follow. In particular, it lets us do away with many excessive baggages like having to model the dynamics of $S(t), h(t, T)$ or $g(t, T)$. It is well known that the commodity futures price volatility is the most tractable quantity in that one can estimate or calibrate it using historical prices or options data. In this sense, it is fair to say that our model exhibits the utmost simplicity.

The next proposition is the key technical result crucial to our approach.

Proposition 2.2. The following results follow from the Model.

(A) (Dynamic of $S(t)$ )

$$
d S_{t}=S_{t} h(t, t) d t+S_{t} \boldsymbol{\sigma}(t, t) \cdot d \mathbf{W}_{t}^{Q}
$$

(B) (Arbitrage-free conditions)

$$
\begin{aligned}
\boldsymbol{\sigma}_{h}(t, T) & =\frac{\partial}{\partial T} \boldsymbol{\sigma}(t, T), \\
\mu_{h}(t, T) & =-\boldsymbol{\sigma}(t, T) \cdot \boldsymbol{\sigma}_{h}(t, T) .
\end{aligned}
$$

Proof. It is easy to see that $F(t, T)$ differentiable with respect to $T$. Since $S_{t}=F(t, t)$, the dynamics of $S(t)=S_{t}$ is easily seen to be

$$
\begin{aligned}
d S(t) & =\frac{\partial F}{\partial T}(t, t) d t+d F(t, t) \\
& =\frac{\partial F}{\partial T}(t, t) d t+S(t) \boldsymbol{\sigma}(t, t) \cdot d \mathbf{W}_{t}^{Q} \\
& =S(t) h(t, t) d t+S(t) \boldsymbol{\sigma}(t, t) \cdot d \mathbf{W}_{t}^{Q}
\end{aligned}
$$

where the last equality is the consequence of (I) of the Model. To prove (B), let

$$
H(t, T)=\int_{t}^{T} h(t, u) d u
$$

Thus

$$
H(t, T)=\int_{t}^{T}\left\{h(0, u)+\int_{0}^{t} \mu_{h}(s, u) d s+\int_{0}^{t} \boldsymbol{\sigma}_{h}(s, u) \cdot d \mathbf{W}_{s}^{Q}\right\} d u .
$$


Using the stochastic Fubini theorem (e.g., Lemma 4.1, p. 116, of [15]), we have

$$
H(t, T)=\int_{t}^{T} h(0, u) d u+\int_{0}^{t} \int_{t}^{T} \mu_{h}(s, u) d u d s+\int_{0}^{t} \int_{t}^{T} \sigma_{h}(s, u) d u \cdot d \mathbf{W}_{s}^{Q} .
$$

Upon differentiating it under the integral sign and collecting terms, we get the following:

$$
\begin{aligned}
d H(t, T) & =-h(t, t) d t+\left(\int_{t}^{T} \mu_{h}(t, u) d u\right) d t+\left(\int_{t}^{T} \boldsymbol{\sigma}_{h}(t, u) d u\right) \cdot d \mathbf{W}_{t}^{Q} \\
& =-h(t, t) d t+M_{h}(t, T) d t+\mathbf{V}_{h}(t, T) \cdot d \mathbf{W}_{t}^{Q}
\end{aligned}
$$

where

$$
\begin{aligned}
& M_{h}(t, T)=\int_{t}^{T} \mu_{h}(t, u) d u, \\
& \mathbf{V}_{h}(t, T)=\int_{t}^{T} \boldsymbol{\sigma}_{h}(t, u) d u
\end{aligned}
$$

Also by the Itô formula,

$$
\begin{aligned}
d e^{H(t, T)}= & e^{H(t, T)}\left(-h(t, t)+M_{h}(t, T)+\frac{1}{2}\left\|\mathbf{V}_{h}(t, T)\right\|^{2}\right) d t \\
& +e^{H(t, T)} \mathbf{V}_{h}(t, T) \cdot d \mathbf{W}_{t}^{Q} .
\end{aligned}
$$

Thus by (A) and (4) above we get

$$
\begin{aligned}
d F(t, T)= & d\left(S(t) e^{H(t, T)}\right) \\
= & S_{t} e^{H(t, T)}\left(M_{h}(t, T)+\frac{1}{2}\left\|\mathbf{V}_{h}(t, T)\right\|^{2}+\boldsymbol{\sigma}(t, t) \cdot \mathbf{V}_{h}(t, T)\right) d t \\
& +S_{t} e^{H(t, T)}\left(\boldsymbol{\sigma}(t, t)+\mathbf{V}_{h}(t, T)\right) \cdot d \mathbf{W}_{t}^{Q} .
\end{aligned}
$$

Comparing the coefficients of $d t$ and $d \mathbf{W}_{t}^{Q}$ of this dynamics with those of the original futures dynamics respectively, we get the following relations:

$$
\boldsymbol{\sigma}(t, t)+\mathbf{V}_{h}(t, T)=\boldsymbol{\sigma}(t, T)
$$

and

$$
M_{h}(t, T)+\frac{1}{2}\left\|\mathbf{V}_{h}(t, T)\right\|^{2}+\boldsymbol{\sigma}(t, t) \cdot \mathbf{V}_{h}(t, T)=0 .
$$

The first formula of (B) follows from differentiating (5) with respect to $T$; the second formula of (B) follows from differentiating (6) with respect to $T$ and making use of (5). 


\section{Volatilities are sufficient model quantities}

In this section, we show how various parts of our Model fit together. In particular, we show that once the interest rate model is given, the whole of our model simply boils down to the single quantity $\boldsymbol{\sigma}$, that is, the commodity futures volatility. The most remarkable consequence of this fact is that all the hard-to-quantify extraneous quantities like convenience yield are implied by the Model, thereby making it unnecessary to calibrate their parameters. We also discuss how the key quantities $\boldsymbol{\sigma}, \boldsymbol{\sigma}_{f}$ and $\boldsymbol{\sigma}_{h}$ are inter-related in such a way that any given two imply the remaining third.

First, comparing the dynamics of $d h(t, T)$ and $d(f(t, T)+g(t, T))$, we have the following relations:

$$
\mu_{f}(t, T)+\mu_{g}(t, T)=\mu_{h}(t, T)=-\boldsymbol{\sigma}(t, T) \cdot \boldsymbol{\sigma}_{h}(t, T)
$$

and

$$
\boldsymbol{\sigma}_{f}(t, T)+\boldsymbol{\sigma}_{g}(t, T)=\boldsymbol{\sigma}_{h}(t, T) .
$$

Thus using Proposition 2.2 and the celebrated HJM drift condition, we can easily see that

$$
\mu_{g}(t, T)=-\boldsymbol{\sigma}(t, T) \cdot \frac{\partial}{\partial T} \boldsymbol{\sigma}(t, T)-\boldsymbol{\sigma}_{f}(t, T) \cdot \int_{t}^{T} \boldsymbol{\sigma}_{f}(t, u) d u
$$

and

$$
\boldsymbol{\sigma}_{g}(t, T)=\frac{\partial}{\partial T} \boldsymbol{\sigma}(t, T)-\boldsymbol{\sigma}_{f}(t, T) .
$$

Therefore in our model the dynamics of $g(t, T)$ is completely determined by $\boldsymbol{\sigma}_{f}(t, T), \boldsymbol{\sigma}(t, T)$ and its $T$-derivative.

On the other hand, Part (B) of Proposition 2.2 says that $\mu_{h}(t, T)$ and $\boldsymbol{\sigma}_{h}(t, T)$ also are determined in a similar fashion. These facts lead to the following theorem.

Theorem 3.1. All the parameters in the Model, hence the Model itself, are completely determined by the forward interest rate volatility $\boldsymbol{\sigma}_{f}(t, T)$ and the commodity futures price volatility $\boldsymbol{\sigma}(t, T)$ and its $T$-derivative $\frac{\partial}{\partial T} \boldsymbol{\sigma}(t, T)$.

Remark 3.2. A word on the $T$-derivative $\frac{\partial}{\partial T} \boldsymbol{\sigma}(t, T)$ of $\boldsymbol{\sigma}(t, T)$ is in order. If $\boldsymbol{\sigma}(t, T)$ is a deterministic function of two variables $t$ and $T, \frac{\partial}{\partial T} \boldsymbol{\sigma}(t, T)$ can be automatically calculated. If $\boldsymbol{\sigma}(t, T)(\omega)$ is a stochastic process of the form $\boldsymbol{\sigma}\left(t, T, X_{t}\right)$ where $\boldsymbol{\sigma}$ is a deterministic function of three variables ( $X_{t}$ could be a vector variable) such that $X_{t}$ is a stochastic process not depending on $T, \frac{\partial}{\partial T} \boldsymbol{\sigma}\left(t, T, X_{t}\right)$ can also be computed by taking the partial derivative with respect to the second variable $T$. So it follows immediately from $\boldsymbol{\sigma}\left(t, T, X_{t}\right)$. However, if $\boldsymbol{\sigma}(t, T)(\omega)$ is a stochastic process depending on another process with $T$ parameter in it, say, if $\boldsymbol{\sigma}\left(t, T, F_{t}^{T}\right)$ is the form of $\boldsymbol{\sigma}$, then its $T$-derivative must 
be taken not only for the second argument $T$ but also with respect to the third argument, thus the $T$-derivative of $F_{t}^{T}$ must enter the picture.

It is also trivial to see that the last equality can be written as

$$
\boldsymbol{\sigma}_{f}(t, T)=\frac{\partial}{\partial T} \boldsymbol{\sigma}(t, T)-\boldsymbol{\sigma}_{g}(t, T),
$$

and the usual HJM drift condition implies that the HJM Model is completely determined by $\boldsymbol{\sigma}_{g}(t, T), \boldsymbol{\sigma}(t, T)$ and its $T$-derivative.

Similarly, integrating (10), we have

$$
\boldsymbol{\sigma}(t, T)-\boldsymbol{\sigma}(t, t)=\int_{t}^{T}\left(\boldsymbol{\sigma}_{f}(t, u)+\boldsymbol{\sigma}_{g}(t, u)\right) d u
$$

It means that $\boldsymbol{\sigma}_{f}(t, T)$ and $\boldsymbol{\sigma}_{g}(t, T)$ determine $\boldsymbol{\sigma}(t, T)$ up to $\boldsymbol{\sigma}(t, t)$.

So far, we have relied on the HJM model to get a handle on the interest rate term structure. But, to our approach, the HJM model is not a must. It is used only because it is most convenient to use in our context. But for our purpose any sensible interest rate model will do. Recall that any sensible interest rate model gives rise to the dynamics of the zero-coupon bond, say,

$$
d P(t, T)=P(t, T)\left[r_{t} d t+\boldsymbol{\sigma}_{P}(t, T) \cdot d \mathbf{W}_{t}^{Q}\right],
$$

where $\boldsymbol{\sigma}_{P}(t, T)$ is the volatility of the zero coupon bond and $r_{t}$ is the short rate of interest. Note that if we were to use the HJM model, $\boldsymbol{\sigma}_{P}(t, T)$ is related to $\boldsymbol{\sigma}_{f}(t, T)$ by

$$
\boldsymbol{\sigma}_{P}(t, T)=-\int_{t}^{T} \boldsymbol{\sigma}_{f}(t, u) d u .
$$

Since it is more common to use in practice the bond price and its volatility, it may be useful to recast what we have discussed in terms of $\boldsymbol{\sigma}_{P}(t, T)$.

First, from (9) and (12), we have

$$
\boldsymbol{\sigma}_{g}(t, T)=\frac{\partial}{\partial T}\left[\boldsymbol{\sigma}(t, T)+\boldsymbol{\sigma}_{P}(t, T) .\right]
$$

Similarly from (8) and (12), we have

$$
\begin{aligned}
\mu_{g}(t, T) & =-\boldsymbol{\sigma}(t, T) \cdot \boldsymbol{\sigma}_{h}(t, T)-\boldsymbol{\sigma}_{f}(t, T) \cdot \int_{t}^{T} \boldsymbol{\sigma}_{f}(t, u) d u \\
& =-\boldsymbol{\sigma}(t, T) \cdot \boldsymbol{\sigma}_{h}(t, T)-\frac{\partial \boldsymbol{\sigma}_{P}}{\partial T}(t, T) \cdot \boldsymbol{\sigma}_{P}(t, T) \\
& =-\frac{1}{2} \frac{\partial}{\partial T}\left[\|\boldsymbol{\sigma}(t, T)\|^{2}+\left\|\boldsymbol{\sigma}_{P}(t, T)\right\|^{2}\right] .
\end{aligned}
$$

Therefore from these, the following analog of Theorem 3.1 is immediate.

Theorem 3.3. All the parameters in the Model, hence the Model itself, are completely determined by the volatility $\boldsymbol{\sigma}_{P}(t, T)$ of the zero-coupon bond and the commodity futures volatility $\boldsymbol{\sigma}(t, T)$ and their respective $T$-derivatives. 


\section{Deriving commodity futures volatility from commodity futures call options}

We have demonstrated so far the overarching importance of the volatility in our Model. The natural question then is how would one go about to fix or calibrate it. The most appealing and natural way is of course to derive the volatility from the option data. In this section, we show how it can be done by following the argument of Dupire [9]. In particular, we will show that once the call option prices are given for all expiry $T$ and all exercise prices $K$, the volatility $\sigma$ must be uniquely expressed.

Let us first fix the delivery date of the futures contract as $T_{1}$ and let $F\left(t, T_{1}\right)$ be the commodity futures price with that delivery date. As Dupire's result posits the stochastic volatility, we also assume that the volatility is of the form $\sigma\left(t, T_{1}, F_{t}^{T_{1}}\right)$ Then, according to our Model, $F\left(t, T_{1}\right)$ satisfies the following:

$$
d F\left(t, T_{1}\right)=\sigma\left(t, T_{1}, F_{t}^{T_{1}}\right) F\left(t, T_{1}\right) d W_{t}^{Q},
$$

where $W_{t}^{Q}$ is a 1-dimensional Brownian motion with respect to the martingale measure $Q$. We choose, for simplicity, to present our result for the onedimensional case; but it is valid for multi-dimension case with virtually no change in proof.

Let $t_{0}<T \leq T_{1}$ and let $F_{0}=F\left(t_{0}, T_{1}\right)$ and $F=F\left(T, T_{1}\right)$. Denote the transition density from $F_{0}$ at time $t_{0}$ to $F$ at time $T$ by $p\left(t_{0}, F_{0} ; T, F\right)$. It is well know that $p\left(t_{0}, F_{0} ; T, F\right)$ satisfies the following Kolmogorov forward equation:

$$
\frac{\partial p}{\partial T}\left(t_{0}, F_{0} ; T, F\right)=\frac{1}{2} \frac{\partial^{2}}{\partial F^{2}}\left(\sigma^{2}\left(T, T_{1}, F\right) F^{2} p\left(t_{0}, F_{0} ; T, F\right)\right) .
$$

Let $C\left(t_{0}, F_{0}, T, K\right)$ be the price of the European call option on $F\left(t, T_{1}\right)$ when the commodity futures price at time $t_{0}$ is $F_{0}=F\left(t_{0}, T_{1}\right)$ with the strike price $K$ at the time of maturity $T$.

Let us assume further that the short rate process $r(t)$ is deterministic. Then it is well known that the call option price is given by:

$$
\begin{aligned}
C\left(t_{0}, F_{0} ; T, K\right) & =e^{-\int_{t_{0}}^{T} r(s) d s} \mathbb{E}^{Q}\left[F\left(T, T_{1}\right)-K\right]^{+} \\
& =e^{-\int_{t_{0}}^{T} r(s) d s} \int_{K}^{\infty}(F-K) p\left(t_{0}, F_{0} ; T, F\right) d F .
\end{aligned}
$$

For convenience later, we define

$$
\begin{aligned}
\tilde{C}\left(t_{0}, F_{0}, T, K\right) & =e^{\int_{t_{0}}^{T} r(s) d s} C\left(t_{0}, F_{0}, T, K\right) \\
& =\int_{K}^{\infty}(F-K) p\left(t_{0}, F_{0} ; T, F\right) d F .
\end{aligned}
$$

As we have alluded at the beginning of this section, we want to derive the $\sigma$ from the option prices. However, to do so, we need to require the call option prices should not vary arbitrarily. For that purpose, we make the assumption that for any given $t_{0}, F_{0}$ and $T, \frac{\partial C}{\partial T}\left(t_{0}, F_{0}, T, K\right) \rightarrow 0$ as $K \rightarrow \infty$. In view of 
the fact $C \rightarrow 0$ as $K \rightarrow \infty$, it is a rather reasonable assumption that meets the expectations of the practitioners. Another assumption we make here is that $\sigma$ is bounded. It is a generally assumed condition for the long-time existence of the solution of the stochastic differential equation of the type (13).

Upon differentiating (15) and making us of (14), we obtain the following result.

Theorem 4.1. Assume that for any given $t_{0}, F_{0}$ and $T, \frac{\partial C}{\partial T}\left(t_{0}, F_{0}, T, K\right) \rightarrow 0$ as $K \rightarrow \infty$ and that $\sigma$ is bounded. If the short rate $r(t)$ is deterministic, then the volatility $\sigma\left(t, T, F_{t}^{T}\right)$ can be derived from call option prices as follows.

$$
\sigma^{2}\left(t, T, F_{t}^{T}\right)=\frac{\frac{\partial C}{\partial T}\left(t_{0}, F_{0}^{T}, t, F_{t}^{T}\right)+r(t) C\left(t_{0}, F_{0}^{T}, t, F_{t}^{T}\right)}{\frac{1}{2}\left(F_{t}^{T}\right)^{2} \frac{\partial^{2} C}{\partial K^{2}}\left(t_{0}, F_{0}^{T}, t, F_{t}^{T}\right)} .
$$

Proof. Twice differentiating (15) with respect to $K$, we get

$$
\frac{\partial^{2} \tilde{C}}{\partial K^{2}}\left(t_{0}, F_{0}, T, K\right)=p\left(t_{0}, F_{0} ; T, K\right) .
$$

Renaming variable $F$ in (14) to $K$, we have

$$
\frac{\partial p}{\partial T}\left(t_{0}, F_{0} ; T, K\right)=\frac{1}{2} \frac{\partial^{2}}{\partial K^{2}}\left(\sigma^{2}\left(T, T_{1}, K\right) K^{2} p\left(t_{0}, F_{0} ; T, K\right)\right) .
$$

Differentiating (16) and making use of (17), we have, after the change of the order of differentiation, that

$$
\frac{\partial^{2}}{\partial K^{2}} \frac{\partial \tilde{C}}{\partial T}\left(t_{0}, F_{0}, T, K\right)=\frac{1}{2} \frac{\partial^{2}}{\partial K^{2}}\left(\sigma^{2}\left(T, T_{1}, K\right) K^{2} p\left(t_{0}, F_{0} ; T, K\right)\right) .
$$

From this we can easily conclude that

$$
\frac{1}{2}\left(\sigma^{2}\left(T, T_{1}, K\right) K^{2} p\left(t_{0}, F_{0} ; T, K\right)\right)=\frac{\partial \tilde{C}}{\partial T}\left(t_{0}, F_{0}, T, K\right)+\alpha K+\beta,
$$

where $\alpha$ and $\beta$ are quantities independent of $K$.

Now it is easy to check that $F=F\left(T, T_{1}\right)$ with the initial condition $F_{0}=$ $F\left(t_{0}, T_{1}\right)$ has a finite second moment, which is given by

$$
\mathbb{E}^{Q}\left[F\left(T, T_{1}\right)\right]=\int K^{2} p\left(t_{0}, F_{0} ; T, K\right) d K .
$$

Therefore $K^{2} p\left(t_{0}, F_{0} ; T, K\right)$ must converge to 0 as $K \rightarrow \infty$. Since $\sigma$ is assumed to be bounded, the left hand side of (18) must converge to 0 as $F \rightarrow \infty$. On the other hand, by the assumption $\frac{\partial \tilde{C}}{\partial T}$ converges to 0 as $F \rightarrow \infty$. Therefore $\alpha$ and $\beta$ must be 0 . Therefore, upon replacing $p$ in (18) with that in (16), we get the following result:

$$
\frac{1}{2} \sigma^{2}\left(T, T_{1}, K\right)=\frac{\frac{\partial \tilde{C}}{\partial T}\left(t_{0}, F_{0}, T, K\right)}{K^{2} \frac{\partial^{2} \tilde{C}}{\partial K^{2}}\left(t_{0}, F_{0}, T, K\right)} .
$$


After we relabel $T$ as $t, T_{1}$ as $T, K$ as $F_{t}^{T}$, and $F_{0}$ as $F_{0}^{T}$, we have

$$
\frac{1}{2} \sigma^{2}\left(t, T, F_{t}^{T}\right)=\frac{\frac{\partial \tilde{C}}{\partial T}\left(t_{0}, F_{0}^{T}, t, F_{t}^{T}\right)}{\left(F_{t}^{T}\right)^{2} \frac{\partial^{2} \tilde{C}}{\partial K^{2}}\left(t_{0}, F_{0}^{T}, t, F_{t}^{T}\right)} .
$$

Upon rewriting this in terms of $C$, the proof is complete.

Acknowledgements. We would like to thank the anonymous referee for many improvements in this paper. In particular, the referee's perceptive comments were instrumental in clearing up several mathematical ambiguities in the earlier version.

\section{References}

[1] L. B. G. Andersen, Markov models for commodity futures: theory and practice, Quant. Finance 10 (2010), no. 8, 831-854.

[2] H. Bessembinder, J. F. Coughenour, P. J. Seguin, and M. M. Smoller, Mean reversion in equilibrium asset prices: evidence from the futures term structure, J. Finance 50 (1995), no. $1,361-375$.

[3] T. Björk, Arbitrage Theory in Continuous Time, Oxford University Press, 2004.

[4] S. Borovkova and H. Geman, Seasonal and stochastic effects in commodity forward curves, Review of Derivatives Research 9 (2006), no. 2, 167-186.

[5] M. J. Brennan, The price of convenience and the valuation of commodity contingent claims, in Lund, D. and Øksendal, B. (eds.), Stochastic Models and Option Values (North-Holland, Amsterdam).

[6] J. Casassus and P. Collin-Dufresne, Stochastic convenience yield implied from commodity futures and interest rates, J. Finance 60 (2005), no. 5, 2283-2331.

[7] J. Casassus, P. Collin-Dufresne, and B. R. Routledge, Equilibrium commodity prices with irreversible investment and non-linear technologies, Available at SSRN: http:// ssrn.com/abstract=686542 or http://dx.doi.org/10.2139/ssrn.686542, 2009.

[8] S. Deng, Stochastic models of energy commodity prices and their applications: meanreversion with jumps and spikes, POWER working papers, Program on Workable Energy Regulation, University of California Energy Institute, 2000.

[9] B. Dupire, Pricing and hedging with smiles, in Mathematics of Derivative Securities (Cambridge, 1995), 103-111, Publ. Newton Inst., 15, Cambridge Univ. Press, Cambridge, 1997.

[10] H. Geman, Commodities and Commodity Derivatives, Wiley, 2005.

[11] H. Geman and A. Roncoroni, Understanding the fine structure of electricity prices, The Journal of Business 79 (2006), no. 3, 1225-1261.

[12] R. Gibson and E. S. Schwartz, Stochastic convenience yield and the pricing of oil contingent claims, J. Finance 15 (1990), no. 3, 959-967.

[13] D. Heath, R. Jarrow, and A. Morton, Bond pricing and the term structure of interest rates: a new methodology for contingent claims valuation, Econometrica 60 (1992), no. $1,77-105$.

[14] J. E. Hilliard and J. Reis, Valuation of commodity futures and options under stochastic convenience yields, interest rates, and jump diffusions in the spot, Journal of Financial and Quantitative Analysis 33 (1998), no. 1, 61-86.

[15] N. Ikeda and S. Watanabe, Stochastic Differential Equations and Diffusion Processes, 2nd Edition, North-Holland Publishing Co., 1989.

[16] R. H. Litzenberger and N. Rabinowitz, Backwardation in oil futures markets: theory and empirical evidence, J. Finance 50 (1995), no. 5, 1517-1545. 
[17] N. T. Milonas, Measuring seasonalities in commodity markets and the half-month effect, Journal of Futures Markets 11 (1991), no. 3, 331-346.

[18] K. R. Miltersen and E. S. Schwartz, Pricing of options on commodity futures with stochastic term structures of convenience yield and interest rates, Journal of Financial Quantitative Analysis 33 (1998), no. 1, 33-59.

[19] V. K. Ng and S. C. Pirrong, Fundamentals and volatility: storage, spreads, and the dynamics of metals prices, The Journal of Business 67 (1994), no. 2, 203-230.

[20] R. S. Pindyck, The dynamics of commodity spot and futures markets: a primer, The Energy Journal 22 (2001), no. 3, 1-30.

[21] D. R. Ribeiro and S. D. Hodges, A two-factor model for commodity prices and futures valuation, EFMA 2004 Basel Meetings Paper.

[22] M. C. Richter and C. Sørensen, Stochastic volatility and seasonality in commodity futures and options: the case of soybeans, EFA 2002 Berlin Meetings Presented Paper.

[23] E. S. Schwartz, The stochastic behavior of commodity prices: implications for valuation and hedging, J. Finance 52 (1997), no. 3, 923-973.

[24] E. Schwartz and J. E. Smith, Short-term variations and long-term dynamics in commodity prices, Management Science 46 (2000), no. 7, 893-911.

[25] Y. Tian and P. Fackler, A seasonal stochastic volatility model for futures price term structure, Working Paper, North California State University, 2000.

[26] A. B. Trolle and E. S. Schwartz, Unspanned stochastic volatility and the pricing of commodity derivatives, EFA 2008 Athens Meetings Paper, 2008.

HyEONG IN CHOI

Department of Mathematics \& Research Institute of Mathematics

Seoul National University

SEOUL 151-747, KOREA

E-mail address: hichoi@snu.ac.kr

SONG-HWA KWON

Department of Mathematics

The Catholic University of Korea

GyeOngGi 420-743, KoreA

E-mail address: skwon@catholic.ac.kr

JUN YEOL KIM

OTC Products Dealing Team

KYOBO SeCurities Co., Ltd

26-4, Yeouido-dong, Yeongdeungpo-gu, Seoul, 150-010, Korea

E-mail address: hustler1@snu.ac.kr

DU-SEOP JUNG

Department of Mathematics

SeOul National University

SEOUL 151-747, KorEA

Current AdDress

Korea Investment \& Securities Co., Ltd.

27-1, Yeouido-dong, Yeongdeungpo-gu, Seoul, 150-745, Korea

E-mail address: duseop.jung@gmail.com 